\title{
TENDENCIAS EN EL USO DE RECURSOS Y HERRAMIENTAS DE LA TECNOLOGÍA EDUCATIVA EN LA EDUCACIÓN UNIVERSITARIA ANTE LA PANDEMIA COVID-19
}

\section{TRENDS IN THE USE OF RESOURCES AND TOOLS OF EDUCATIONAL TECHNOLOGY IN UNIVERSITY EDUCATION IN THE FACE OF THE COVID-19 PANDEMIC}

\author{
Sergio Junior Navarro-Hudiel ${ }^{1}$
}

(Recibido/received: 20-octubre-2020; aceptado/accepted: 4-diciembre-2020)

RESUMEN: La pandemia del COVID-19 ha sido un hecho sin precedentes que ha marcado un antes y un después para toda la humanidad. La educación universitaria, no es ajena a estos cambios, surgiendo la necesidad adaptarse ante el contexto y tendencias en el uso de las tecnologías educativas acompañadas de estrategias didácticas y pedagógicas para alcanzar aprendizajes significativos y fortalecer los procesos de enseñanza aprendizaje complementarias a las clases presenciales. Dado que la tecnología representa los procedimientos y técnicas, que de manera ordenada representan una herramienta que contribuye a resolver situaciones aplicando la ciencia, en este artículo se hace referencia a prácticas educativas desarrolladas por instituciones de educación superior a nivel regional y latinoamericano para aprovechar los recursos y herramientas que la tecnología educativa dispone y que son de uso común en los miembros de la comunidad educativa. De igual manera, se describen las herramientas y recursos empleados por la comunidad educativa, complementarios a las clases presenciales, desarrolladas en la Universidad Nacional de Ingeniería, Sede Regional UNI Norte tanto de los docentes, así como los estudiantes. Se plantea reflexiones y posibles estudios vinculados a la calidad educativa y el uso de experiencias exitosas que contribuyan a la mejora de procesos de enseñanza aprendizaje.

PALABRAS CLAVE: Tecnología educativas, Recursos y herramientas, calidad educativa

\footnotetext{
1 Universidad Nacional de Ingeniería Estelí, Nicaragua. Correo: Sergio.Navarro@norte.uni.edu.ni, Orcid:
} https://orcid.org/0000-0002-7001-5860 
ABSTRACT: The COVID-19 pandemic has been an unprecedented event that has marked a before and after for all humanity. University education is not excluded to these changes, emerging the need to adapt to the context and trends in the use of educational technologies accompanied by didactic and pedagogical strategies to achieve significant learning and strengthen the teachinglearning processes complementary to face-to-face classes. Given that technology represents procedures and techniques, which in an orderly manner represent a tool that contributes to solving situations by applying science, this article refers to educational practices developed by higher education institutions at the regional and Latin American level to take advantage of resources and tools that educational technology has available and that are commonly used by members of the educational community. In the same way, the tools and resources used by the educational community are described, complementary to the face-to-face classes, developed at the National University of Engineering, UNI North Regional Headquarters for both the teachers and the students. Reflections and possible studies related to educational quality and the use of successful experiences that contribute to the improvement of teaching-learning processes are proposed.

KEYWORDS: Educational technology, Resources and tools, educational quality

\section{INTRODUCCIÓN}

Como refiere la Organización para la cooperación económica y el desarrollo - OCDE (2020) y Ayuso, Requena, Jiménez y Khamis (2020) la pandemia de la COVID-19 ha marcado un punto de cambio en la vida de todos los individuos del planeta, cambiando la forma en que se aprende y se trabaja. En respuesta ante la pandemia el aprendizaje digital se ha vuelto clave para que los estudiantes continúen desarrollando sus competencias y habilidades para la vida, siendo las universidades han implementado soluciones digitales para afrontar la crisis (p.8). De igual manera plantean García, Reyes y Godínez (2018) que las Tecnologías de la Información y Comunicación en la educación superior representan nuevos entornos y oportunidades de aprendizaje individual y colectivo, que, considerando su impacto en la educación, son desarrolladoras de competencias y habilidades necesarias para el aprendizaje, así como desarrollo de habilidades para el desempeño de los estudiantes en la sociedad.

Con el objetivo de identificar y dar a conocer herramientas y recursos empleados en la educación superior como complemento al desarrollo de clases presenciales, así como identificar aquellos que los docentes y estudiantes de la comunidad educativa de Sede Regional UNI Norte utilizan, fue necesario realizar un análisis de información recopilada mediante la aplicación de encuestas, así como análisis documental para establecer líneas base para continuar fortaleciendo la tecnología educativa en pro de la mejora de los procesos de enseñanza aprendizaje. Sin duda, son muchas las herramientas y recursos dispuestos por la tecnología educativa para ser un componente complementario al desarrollo de las clases. De manera general, acorde Álvarez et al. (2020), VIU (2018), Cruz, Pozo V, Andino y Arias (2018), estas podrán ser categorizadas en cuatro grupos correspondientes a colaboración, creación de blogs, para comunicación y para creación de entornos de trabajo.

El Higo Revista Científica / Volumen 10. No. 01, pp. 111-122 / diciembre 2020 


\section{METODOLOGÍA}

Acorde a Hernández, Fernández y Baptista (2014) esta investigación es de tipo descriptiva con un enfoque filosófico Mixto. Para la evaluación de las tendencias en adopción de herramientas TIC's para apoyar la labor docente se realizó un análisis documental basado en artículos del último año del cual se realizó una síntesis integradora en las tendencias. Se empleó base de datos nacional dispuesta por el Consejo Nacional de Universidades (CNU), así como otros recursos encontrados en la búsqueda avanzada de los últimos tres años. Para el diagnóstico de las herramientas base conocidas en la comunidad docentes de la Sede Regional del Norte de la Universidad Nacional de Ingeniería, se desarrolló una encuesta para los docentes de 39 asignaturas, apoyadas por Google formularios, en la cual los involucrados en una escala dicotómica (sí o no) describieron las herramientas que apoyan su práctica docente con las clases presenciales, de igual manera, se organizó una encuesta estructurada, la cual fue aplicada con 93\% de confianza, Munch, L., y Angeles, E. (1996) aplicando el criterio de población finita para una población estudiantil de 590 estudiantes, obteniendo una muestra de 173 datos muestrales de los miembros de la comunidad estudiantil de la Sede Regional del Norte de la Universidad Nacional de Ingeniería, UNI, Nicaragua.

\section{RESULTADOS Y DISCUSIÓN}

En correspondencia con Sandoval (2020), Álvarez et al. (2020), Tejedor, Cervi, Tusa y Parola (2020) los maestros han adoptado herramientas en su práctica docente a fin de fortalecer los procesos de enseñanza aprendizaje, demostrando la necesidad de actualizar conocimientos en los aspectos pedagógicos, tecnológicos y didácticos. De igual manera, los espacios como aulas virtuales son empleados como alternativas complementarias a la docencia directa haciendo un cambio ante las necesidades de aprovechamiento de plataformas, tal y como lo refieren autores como Vergel (2020), Nuguer y Powell (2020), de Luca (2020), Crespo y Palaguachi (2020) y Álvarez et al. (2020). En oposición a resultados de otros estudios como Arancibia, Cabero y Marín (2020) y en lineamiento con planteamientos de Laro (2020), Espinel (2020), Medina, Lagunes y Guerra (2020) y Gutiérrez, Virgilio, Moreno y Maruri (2020) en la Universidad Nacional de Ingeniería, Sede Regional del Norte, se cuenta con una apertura a nuevos conocimientos y una actitud positiva para complementar la formación presencial con recursos TIC's promoviendo innovaciones pedagógicas generando espacios para las actualizaciones complementarias y continuas en la tecnología educativa, destacando ventajas en el uso de estos recursos y herramientas que la tecnología educativa permite.

Según Molinero y Chávez (2019), el dispositivo que más utilizan los estudiantes en la escuela es la computadora, y las aplicaciones más usadas allí son Drive, Facebook y YouTube. En cuanto a las redes sociales, las más sobresalientes son Facebook, YouTube e Instagram (Ver figura 4). Las aplicaciones más utilizadas en el teléfono celular, mientras tanto, son WhatsApp, Facebook y YouTube. Los programas de Microsoft Office siguen siendo los preferidos por los estudiantes, ya que el procesador de texto que más se utiliza es Word, seguido del procesador de texto en 
línea. Para los ejercicios estadísticos, el más utilizado es Excel. En lo que se refiere a las presentaciones, el que más sobresale es PowerPoint, seguido de Prezzi.

Las preferencias de los estudiantes para el desarrollo de sus clases en relación a la plataforma o recurso educativo se muestran en la figura 1 , lo cual es complementario con los sitios para administrar recursos y respaldar información mostrado en la figura 2.

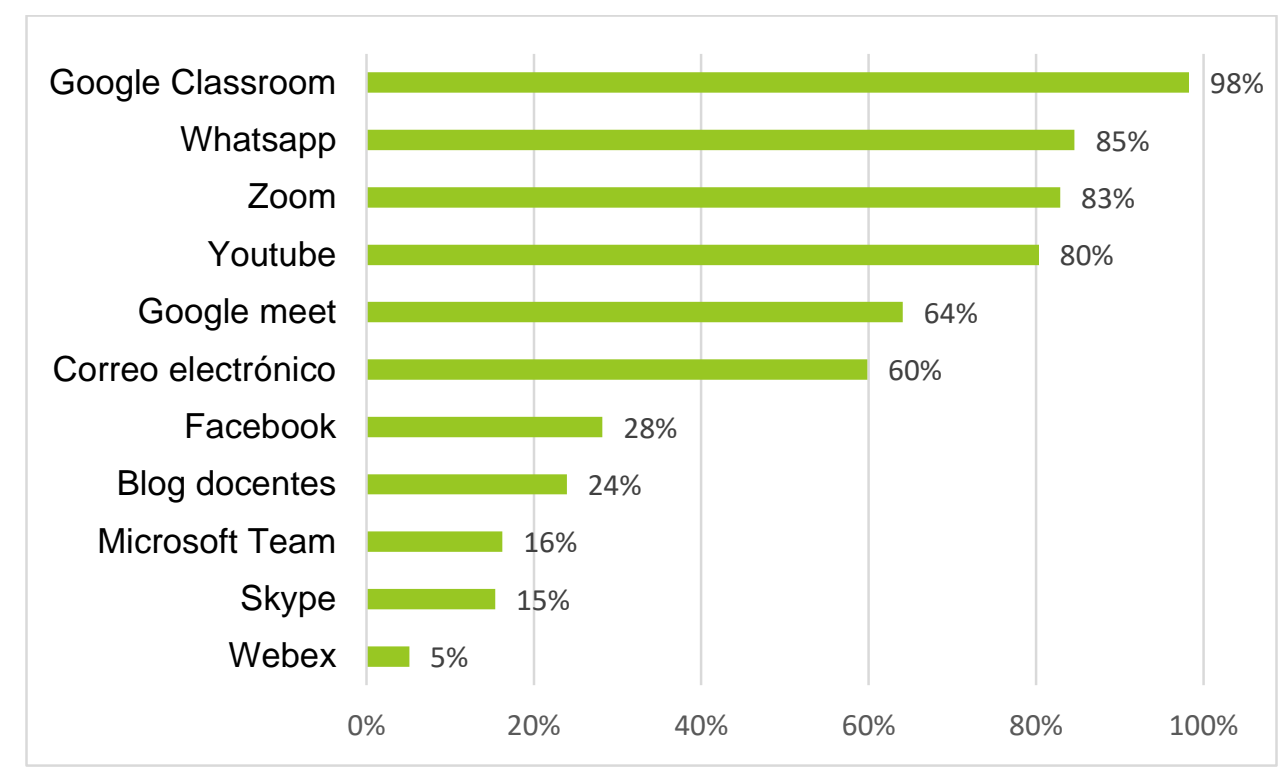

Figura 1. Preferencia como plataforma o recurso educativo.

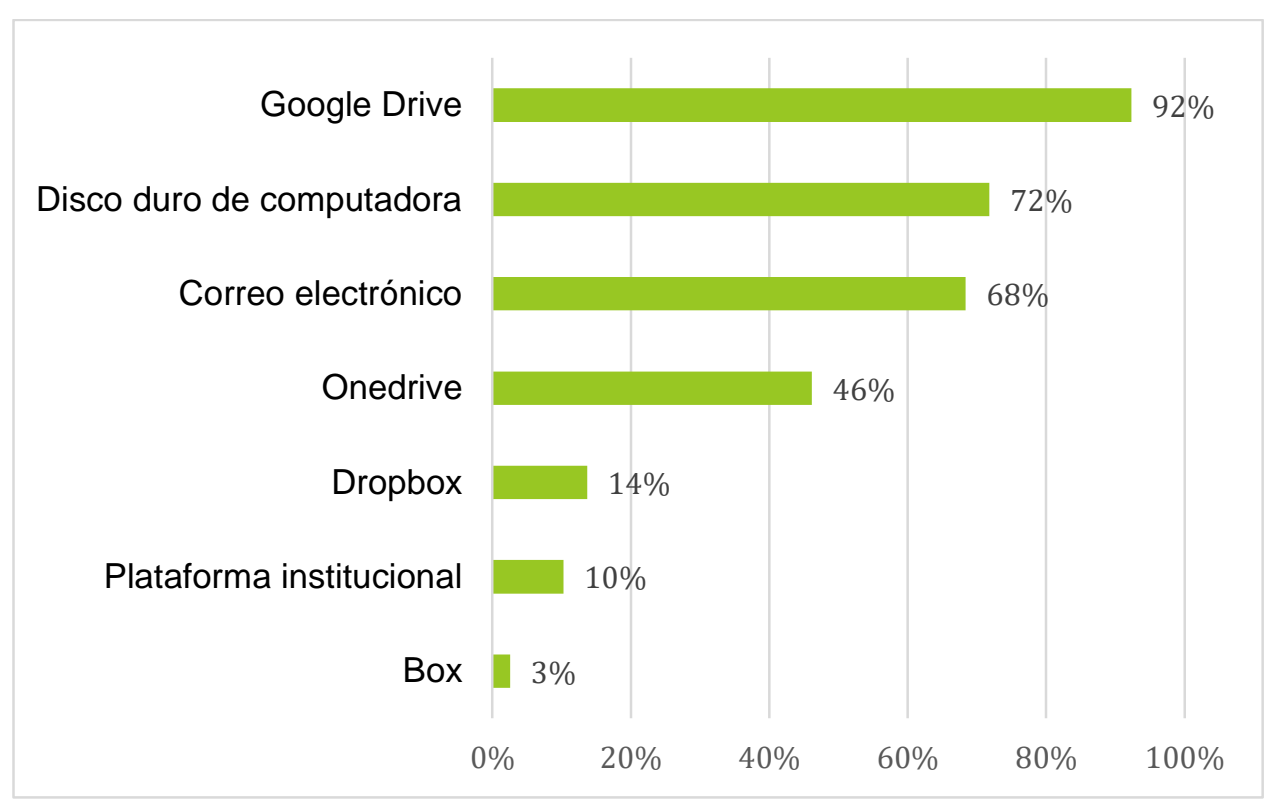

Figura 2. Sitios de administración de información usada por los estudiantes

El Higo Revista Científica / Volumen 10. No. 01, pp. 111-122 / diciembre 2020 
Las redes sociales más usadas en la comunidad educativa de la Sede Regional son las mostradas en la figura 3.

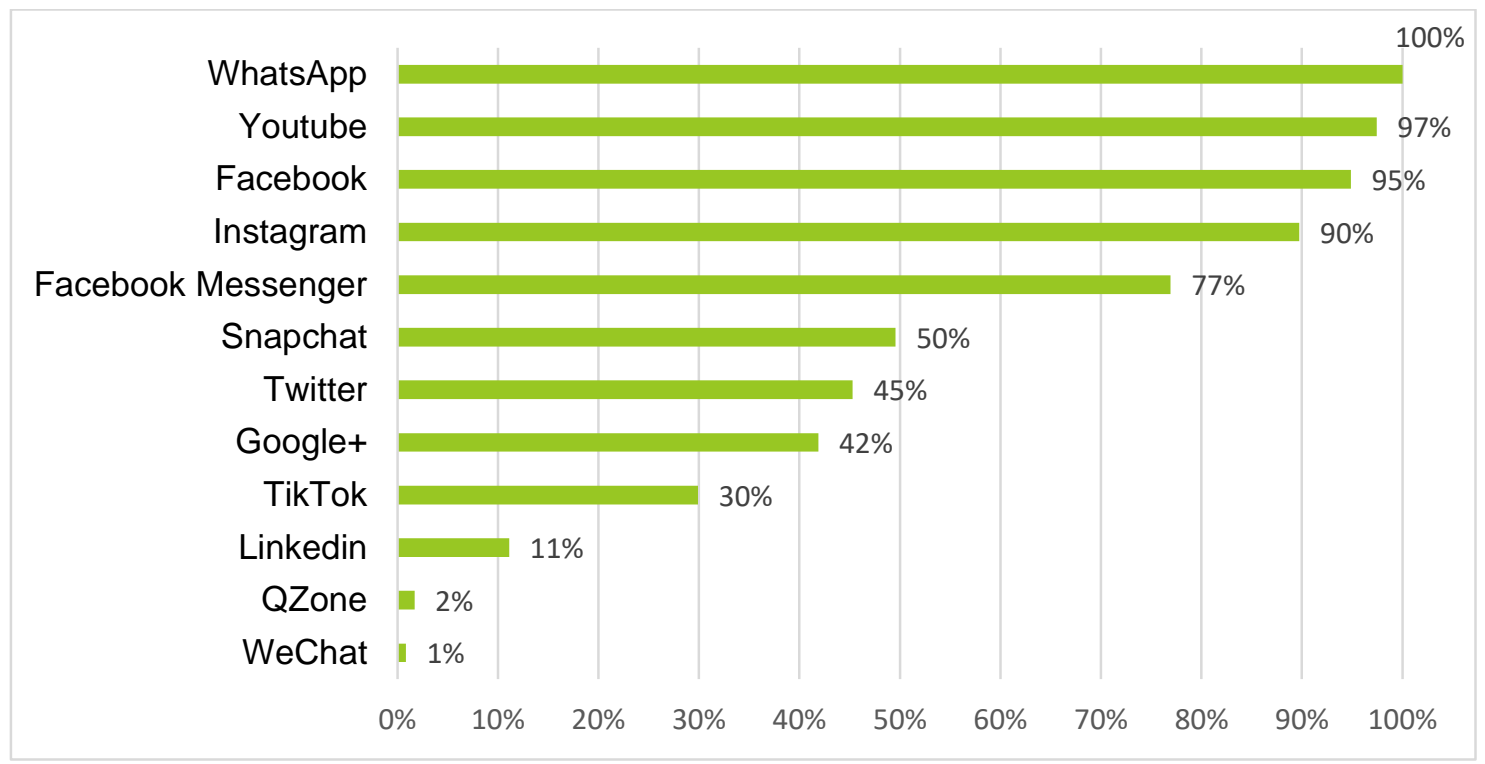

Figura 3. Redes sociales empleadas por estudiantes

La comunidad educativa docente conoce y aplica herramientas TIC's, que facilitan el proceso de enseñanza aprendizaje. Se hace uso de los recursos de fácil acceso y practicidad. Los recursos más empleados para este proceso son el Google Classroom, los video-tutoriales, las herramientas del entorno de Office (Microsoft Word, Microsoft PowerPoint y Microsoft Excel) incluyendo a Google Chrome como principal motor de búsqueda en la internet, figura 4.

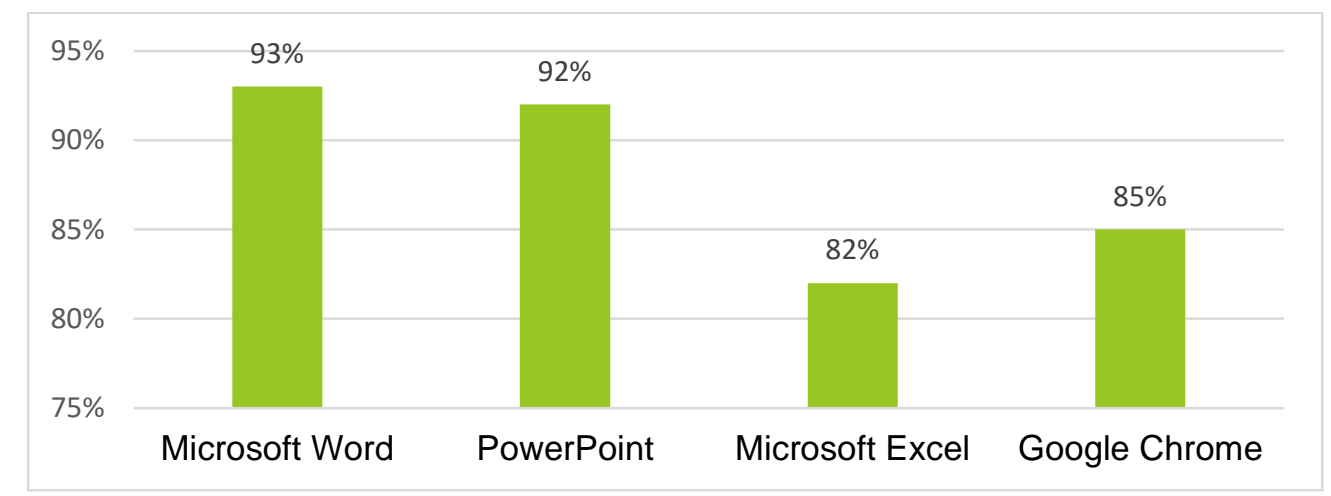

Figura 4. Principales recursos empleados como procesador de texto, elaborador de presentaciones interactivas, hojas de cálculo y motor de búsqueda.

Además de las herramientas digitales empleadas por los docentes de la comunidad educativa de Sede Regional UNI Norte, que complementa la docencia directa mostrada en tabla 1, para los miembros de la comunidad educativa de la Sede Regional UNI Norte, como competencias transversales y conocimientos también se hace uso de otros recursos y programas específicos

El Higo Revista Científica / Volumen 10. No. 01, pp. 111-122 / diciembre 2020 
que fortalecen los procesos de enseñanza aprendizaje recomendados por los autores Vaillant, Zidán y Biagas (2020), Venegas, Luzardo y Pereira (2020), Paz y Gisbert (2020), Mayorga (2020), Islas (2018) y Varela y Valenzuela (2020). Entre estos recursos se destacan las plataformas TEAM, Zoom, EVA, OBS, Page, Note, Látex, llamadas telefónicas, INSHOT, Discord, Filmora Wondershare, SAP2000, Autocad, Global Mapper, QGIS, Pixton, Plataforma Web, YouTube, rollApp, formularios de Google, Idoceo Connet, Aplicaciones online, MINDOMO y otras aplicaciones para teléfonos móviles, tablets así como las computadoras. Las herramientas, se muestra en la tabla 1.

Tabla 1. Herramientas TIC's empleadas por docentes complementarias a clases presenciales

\begin{tabular}{lc}
\hline Recurso empleado & $\begin{array}{c}\text { Porcentaje de docentes que } \\
\text { lo emplean complementario } \\
\text { a sus clases }\end{array}$ \\
\hline Classroom & 100 \\
WhatsApp & 97 \\
Herramientas de Microsoft & 92 \\
Office & \\
Video Tutoriales & 90 \\
Herramientas de Google & 87 \\
Correo electrónico & 74 \\
Reuniones Virtuales con & 57 \\
Zoom & \\
Messenger & 33 \\
Herramientas de & 8 \\
gamificación & \\
Facebook Live & 3
\end{tabular}

Se propone el desarrollo de una guía uniforme, acorde a lineamientos de cada institución educativa, para el desarrollo de las tareas o asignaciones de las clases, estructura en cuatro secciones, en la cual se detallen las generalidades del tema, objetivos, procedimiento y evaluación de actividades, incluyendo recursos de base y referencia adicional. La comunidad educativa también innova en el uso de nuevas herramientas y recursos, que tienden a ser actualizadas surgiendo con el objetivo de apoyar la labor docente.

Una estructura propuesta para fomentar la integración de aprendizajes y el uso complementario de TIC'S en la docencia que podría ser empleada como referente se detalla a continuación:

Datos generales: Asignatura, Grupo, nombre de Unidad temática, Nombre del Tema a desarrollar y fecha de asignación: 
I.- Presentación e importancia del contenido a desarrollar

Se realiza una breve referencia relacionada con el contenido a desarrollar en esta asignación (sesión semipresencial) destacando la importancia del tema y aclare a estudiante el contenido a desarrollar.

II.- Objetivos de aprendizaje de la actividad

Se indican los objetivos que se desean alcanzar desde las perspectiva y aprendizajes de los estudiantes para esta actividad. Los objetivos deberán de ser alcanzables y evidenciables en esta asignación y podrán tener al menos tres elementos sugeridos tales como Verbo + objeto + cómo + condición o criterio de calidad. De ser posible se sugiere plasmar los objetivos tanto conceptuales, procedimentales y actitudinales.

\section{Orientaciones de actividad a realizar}

Se incluyen de forma descriptiva con qué recursos didácticos el estudiante hace la actividad de aprendizaje. Se describen los recursos disponibles de apoyo para tal tarea. Se establece con claridad la comunicación por los medios tecnológicos disponibles para motivar, aclarar o sugerir al estudiante sobre la actividad de aprendizaje que realizan.

Se solicita detallar la forma en que se organizará esta actividad de aprendizaje acorde a material proporcionado. Definir organización de la entrega (Individual, colectiva, estructura y fecha de entrega, etc.)

Se detalla lo mejor posible el paso a paso las actividades del estudiante a realizar para esta actividad.

Es importante disponer a alcance de estudiantes por redes sociales, correo, WhatsApp o en plataforma de Google Classroom (o Google Drive) así como espacios de blog: Material de lectura base (elaborado o compilados por su persona) para tal actividad. Enlaces a material complementario, videotutoriales o sitios web de apoyo (validando su utilidad).

Se sugiere usar diferentes estrategias de evaluación en las actividades indicadas. Considerando la cantidad de asignatura que el estudiante cursa, se sugiere realizar una asignación por semana otorgando toda la semana para su resolución y envío.

\section{Evaluación}

Se sugiere aclarar que esta asignación es semipresencial y tiene un peso ponderado de la nota final. Es necesario indicar el tipo de evaluación (formativa o sumativa) en el proceso de formación del estudiante. 
En cuanto a aspectos de forma o formato es recomendable que el docente deje evidencias de sus propias creatividades e innovaciones educativas con elementos gráficos que fortalezcan el logro del aprendizaje en sus estudiantes.

Podrá indicar los criterios de evaluación. Opcionalmente podrá crear una matriz de indicadores de evaluación (Rúbrica).

\section{CONCLUSIONES}

En la actualidad la posición de las Universidades es brindar las condiciones a su claustro de docentes para que se den la oportunidad de incursionar en el mundo virtual acercando al estudiante con sus facilitadores, acortando distancias, permitiendo que el conocimiento no experimente barreras que interrumpan el verdadero objetivo, el verdadero quehacer de la enseñanza. Hoy la Comunidad Universitaria se enfrenta a una terrible situación a la que todos llaman Pandemia, pero la educación no debe sentirse amenazada en ningún momento, deben fluir los procesos, debe llegar ese conocimiento tan anhelado y necesario por los estudiantes, por la Sociedad.

Este momento es simplemente un impase del cual la Educación tiene que salir victoriosa y fortalecida, con mejores competencias, con estudiantes convencidos de sus capacidades, al mencionar la otra parte de este binomio perfecto, no sólo el docente trasciende su zona de confort, ¿dónde se deja al estudiante?, estudiantes rurales, de escasos recursos pero son ellos los más preocupados por continuar con sus estudios, son quienes se preocupan por su formación y por continuar liberándose de temores a lo desconocido, en este caso la Tecnología, el mito de la educación utilizando herramientas TIC.

Estos procesos por más recursos que se tengan no son fáciles, la educación presencial convencional ya no será la misma después de la Pandemia del COVID 19, muchos docentes familiarizados con nuevas o ya conocidas herramientas TIC optarán por continuar sus procesos de enseñanza aprendizaje haciendo uso de las mismas, y los estudiantes quienes se preparan integralmente mejorarán cada vez más y harán de ellas su cotidianidad. Es necesario profundizar en estudios detallados en relación al uso de la tecnología retomando lo planteado por expertos en la temática. Tal y como refiere Toribio (2016) son muchas las experiencias de uso de TIC's en las aulas de clases. Algunas muestran que los procesos de aprendizaje mejoran y que los estudiantes desarrollan diferentes habilidades derivadas del uso de la tecnología. Otras, en cambio, muestran que el uso de los computadores no incrementa los aprendizajes, por el contrario, genera obstáculos relacionados con las actitudes hacia la tecnología y con las estrategias usadas para su incorporación. En perspectivas planteadas por Wenczenovicz (2020), Jordá y López (2020) es necesario superar dificultades en el uso de las herramientas TIC's que permitan fortalecer los procesos de enseñanza. 
En concordancia con Pastran, Gil y Cervantes (2020), De la Hoz, Martínez, Combita y Hernández (2019), Guillen, Herrera y Ale de la Rosa (2018) y García et al. (2018) las tecnologías de la información son un complemento al desarrollo de clases presenciales que permitirán ampliar la interacción, el autoaprendizaje y las habilidades de búsqueda de información aprovechando el tiempo y recursos institucionales y familiares. Como indica Monroy (2020), Islas (2018) y Abarca (2015) el dominio de las tecnologías y recursos digitales es una habilidad necesaria para los docentes que les permita comprender, seleccionar y adecuar las herramientas a su disposición de manera que sus estudiantes alcances los objetivos y/o competencias previstas en el desarrollo de los cursos en consecución de los procesos evolutivos de su adopción e implementación que incluyen el acceso, adopción, adaptación, apropiación invención ya referidos por Pedro (2020), Álvarez et al. (2020), Beatriz, Luzardo y Aguilar (2019) y Salmerón (2018), UNESCO (2017), Puentes (2017) y Alderete, Jones y Morero (2014).

\section{REFERENCIAS}

Abarca, Y. (2015). El uso de las TIC en la educación universitaria: motivación que incide en su uso y frecuencia. Revista de Lenguas Modernas, (22). https://doi.org/10.15517/rlm.v0i22.19692

Alderete, M., Jones, C. y Morero, H. (2014). Explanatory factors of ICT adoption in the automotive and steel industries in Argentine. Revista Científica Pensamiento y Gestión, 37, 1-40. https://doi.org/10.14482/pege.37.7019

Álvarez, H., Arias, E., Bergamaschi, A., López Sánchez, Á., Noli, A., Ortiz, M., ... Viteri, A. (2020). La educación en tiempos del coronavirus: Los sistemas educativos de América Latina y el Caribe ante COVID-19. Washington, D.C. https://doi.org/10.18235/0002337

Arancibia, M., Cabero, J. y Marín, V. (2020). Creencias sobre la enseñanza y uso de las tecnologías de la información y la comunicación (TIC) en docentes de educación superior. Formación Universitaria, 13(3), 89-100. https://doi.org/10.4067/S0718-50062020000300089

Ayuso, L., Requena, F., Jiménez, O. y Khamis, N. (2020). The Effects of COVID-19 Confinement on the Spanish Family: Adaptation or Change? Journal of Comparative Family Studies, 51(34), 274-287. https://doi.org/10.3138/jcfs.51.3-4.004

Beatriz, S., Luzardo, M. y Aguilar, A. (2019). Apropiación de las Tecnologías de Información y Comunicación como Generadoras de Innovaciones Educativas. Ciencia, Docencia y Tecnología, (Vol30No58). https://doi.org/10.33255/3058/413

Crespo, M. y Palaguachi, M. (2020). Educación con Tecnología en una Pandemia: Breve Análisis. Revista Scientific, 5(17), 292-310. https://doi.org/10.29394/Scientific.issn.25422987.2020.5.17.16.292-310

Cruz, M., Pozo V, M. A., Andino, A. y Arias, A. (2018). Las Tecnologías de la Información y la Comunicación (TIC) como forma investigativa interdisciplinaria con un enfoque intercultural para el proceso de formación de los estudiantes. E-Ciencias de La Información.

El Higo Revista Científica / Volumen 10. No. 01, pp. 111-122 / diciembre 2020 
https://doi.org/10.15517/eci.v1i1.33052

De-la-Hoz, E., Martínez, O., Combita, H. y Hernández, H. (2019). Las Tecnologías de la Información y la Comunicación y su Influencia en la Transformación de la Educación Superior en Colombia para Impulso de la Economía Global. Información Tecnológica, 30(1), 255-262. https://doi.org/10.4067/S0718-07642019000100255

de Luca, M. (2020). Las aulas virtuales en la formación docente como estrategia de continuidad pedagógica en tiempos de pandemia. Usos y paradojas. Análisis Carolina. https://doi.org/10.33960/AC_33.2020

Espinel, E. (2020). La tecnología en el aprendizaje del estudiantado de la Facultad de Ciencias Químicas, Universidad Central del Ecuador. Actualidades Investigativas En Educación, 20(2), 1-39. https://doi.org/10.15517/aie.v20i2.41653

García, M., Reyes, J. y Godínez, G. (2018). Las Tic en la educación superior, innovaciones y retos / The ICT in higher education, innovations and challenges. RICSH Revista Iberoamericana de Las Ciencias Sociales y Humanísticas, 6(12), 299-316. https://doi.org/10.23913/ricsh.v6i12.135

Guillen, L., Herrera, A. P. y Ale de la Rosa, Y. (2018). Las herramientas tecnológicas TIC's como elemento alternativa para el desarrollo del componente físico (ICT technological tools as an alternative element for the development of the physical component). Retos, (34), 222-229. https://doi.org/10.47197/retos.v0i34.60044

Gutiérrez, R., Virgilio, V., Moreno, N. y Maruri, C. (2020). Herramientas pedagógicas innovadoras en el Recinto "Urania Montás", San Juan de la Maguana, República Dominicana. International Journal of New Education, 3(1). https://doi.org/10.24310/IJNE3.1.2020.8511

Hernández, R., Fernández, C. y Baptista, M. (2014). Metodología de la investigación (Sexta). Mexico: Mc Graw Hill. Recuperado de https://trabajosocialudocpno.files.wordpress.com/2017/07/metodologc3a3c2ada_de_la_inv estigacic3a3c2b3n_-sampieri-_6ta_edicion1.pdf

Islas, C. (2018). La implicación de las TIC en la educación: Alcances, Limitaciones y Prospectiva / The role of ICT in education: Applications, Limitations, and Future Trends. RIDE Revista Iberoamericana Para La Investigación y El Desarrollo Educativo, 8(15), 861-876. https://doi.org/10.23913/ride.v8i15.324

Jordá, R. y Lopez, J. (2020). Factores de crecimiento económico en los países en desarrollo: el papel de las TICs. Boletín de La Asociación de Geógrafos Españoles, (86). https://doi.org/10.21138/bage.2979

Laro, E. (2020). Innovar enseñando: la educación del futuro. Las TICs como factor motivador en la enseñanza. REJIE Revista Jurídica de Investigación e Innovación Educativa. https://doi.org/10.24310/REJIE.2020.v0i21.7530

Mayorga, M. (2020). Conocimiento, aplicación e integración de las TIC - TAC y TEP por los docentes universitarios de la ciudad de Ambato. Revista Tecnológica-Educativa Docentes 
2.0, 9(1), 5-11. https://doi.org/10.37843/rted.v9i1.101

Medina, H., Lagunes, A. y Guerra, M. T. (2020). ¿Qué aportan las Tecnologías de la Información y Comunicación en la enseñanza de las ciencias? Revista Digital Universitaria, 21(3). https://doi.org/10.22201/codeic.16076079e.2020.v21n3.a9

Munch, L., y Angeles, E. (1996). Método y técnicas de Investigación. México: Trillas. Página 102.

Molinero, M. y Chávez, U. (2019). Herramientas tecnológicas en el proceso de enseñanzaaprendizaje en estudiantes de educación superior. RIDE Revista Iberoamericana Para La Investigación y El Desarrollo Educativo, 10(19). https://doi.org/10.23913/ride.v10i19.494

Monroy, G. (2020). Herramientas tecnológicas aplicadas a la educación a distancia. Retrieved November 26, 2020, from https://www.gicesperu.org/articulo.php?id=q+sNp2eAe7ON4EYpqsMuAQ==

Nuguer, V. y Powell, A. (2020). 2020 Latin American and Caribbean Macroeconomic Report: Policies to Fight the Pandemic. Washington, D.C. https://doi.org/10.18235/0002284

OCDE. (2020). Making the Most of Technology for Learning and Training in Latin America. OECD. https://doi.org/10.1787/ce2b1a62-en

Pastran, M., Gil, A. y Cervantes, D. (2020). En tiempos de coronavirus: las TIC'S son una buena alternativa para la educación remota. Revista Boletín Redipe, 9(8), 158-165. https://doi.org/10.36260/rbr.v9i8.1048

Paz, L. y Gisbert, M. (2020). Desafíos para las universidades colombianas frente a políticas nacionales e internacionales de integración de TIC en la educación. Edutec. Revista Electrónica de Tecnología Educativa, (73), 51-65. https://doi.org/10.21556/edutec.2020.73.1617

Pedró, F. (2020). COVID-19 y educación superior en América Latina y el Caribe: Efectos, impactos y recomendaciones políticas. Análisis Carolina. https://doi.org/10.33960/AC_36.2020

Puentes, R. (2017). Análisis de la apropiación y uso de las TIC por parte de las pymes colombianas. IUSTA, 1(46). https://doi.org/10.15332/s1900-0448.2017.0046.01

Salmerón, A. (2018). Las TIC's en la educación. Retrieved November 26, 2020, from https://medac.es/blogs/educacion-infantil/las-herramientas-tic-en-la-educacion/

Sandoval, C. (2020). La Educación en Tiempo del Covid-19 Herramientas TIC: El Nuevo Rol Docente en el Fortalecimiento del Proceso Enseñanza Aprendizaje de las Prácticas Educativa Innovadoras. Revista Tecnológica-Educativa Docentes 2.0, 9(2), 24-31. https://doi.org/10.37843/rted.v9i2.138

Tejedor, S., Cervi, L., Tusa, F. y Parola, A. (2020). Educación en tiempos de pandemia: reflexiones de alumnos y profesores sobre la enseñanza virtual universitaria en España, Italia y Ecuador. Revista Latina, (78), 1-21. https://doi.org/10.4185/RLCS-2020-1466 
Toribio, J. (2016). Home " Notas de Prensa " Las TICs en las universidades del CNU Tics Las TICs en las universidades del CNU. Retrieved November 26, 2020, from http://www.cnu.edu.ni/las-tics-en-las-universidades-del-cnu/

UNESCO. (2017). TIC, educación y desarrollo social en América Latina y el Caribe. Montevideo. Recuperado de https://unesdoc.unesco.org/ark:/48223/pf0000262862

Vaillant, D., Zidán, E. y Biagas, G. (2020). Uso de plataformas y herramientas digitales para la enseñanza de la Matemática. Ensaio: Avaliação e Políticas Públicas Em Educação, 28(108), 718-740. https://doi.org/10.1590/s0104-40362020002802241

Varela, S. y Valenzuela, J. (2020). Uso de las tecnologías de la información y la comunicación como competencia transversal en la formación inicial de docentes. Revista Electrónica Educare, 24(1), 1-20. https://doi.org/10.15359/ree.24-1.10

Venegas, L., Luzardo, H. y Pereira, A. (2020). Conocimiento, formación y uso de herramientas TIC aplicadas a la Educación Superior por el profesorado de la Universidad Miguel de Cervantes. Edutec. Revista Electrónica de Tecnología Educativa, (71), 35-52. https://doi.org/10.21556/edutec.2020.71.1405

Vergel, M. (2020). Educación, Covid y TIC. Revista Boletín Redipe, 9(8), 18-23. https://doi.org/10.36260/rbr.v9i8.1037

VIU. (2018). 15 herramientas TIC para colaborar con compañeros y profesores. Retrieved November 26, 2020, from https://www.universidadviu.com/15-herramientas-tic-colaborarcompaneros-profesores/

Wenczenovicz, T. (2020). Ensino a distância, dificuldades presencias: perspectivas em tempos de COVID-19. Revista Ibero-Americana de Estudos Em Educação, 15(4), 1750-1768. https://doi.org/10.21723/riaee.v15i4.13761

\section{SEMBLANZA DE AUTOR}

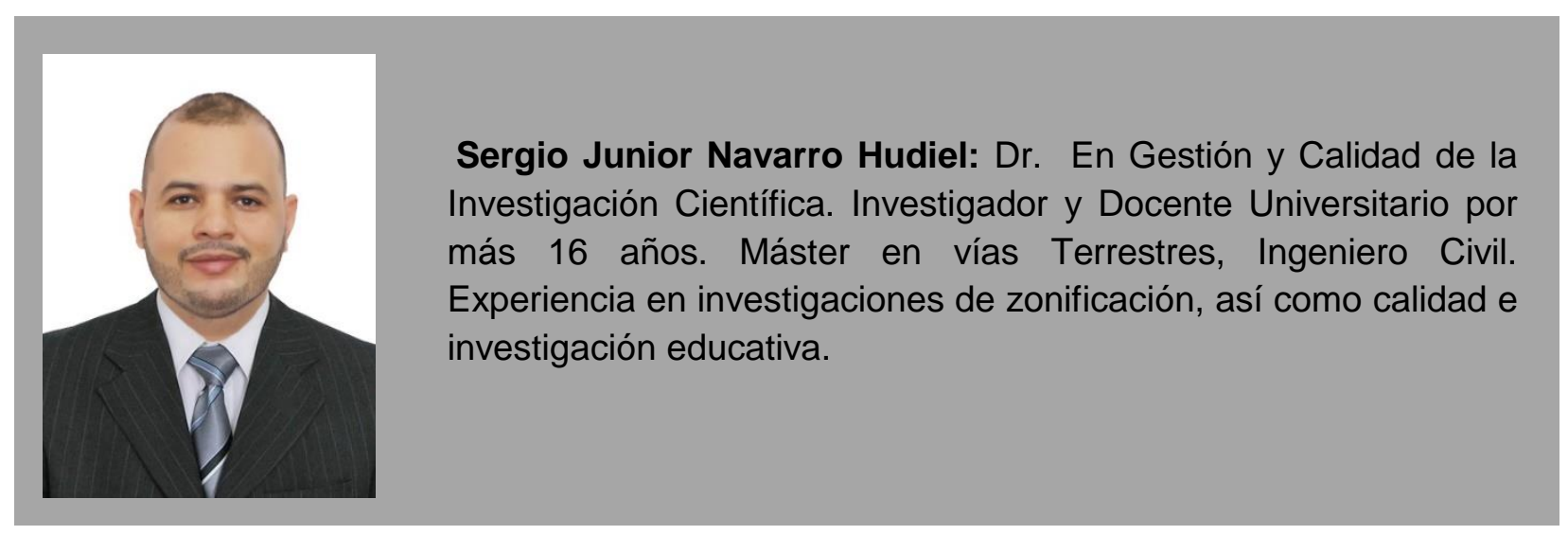

